\title{
A novel homozygous nonsense mutation (p.Y78*) in TMPRSS6 gene causing iron-refractory iron deficiency anemia (IRIDA) in two siblings
}

\author{
Seda Çakmakl1 ${ }^{1}$, Çiğgdem Kaplan², Mehmet Uzunoğlu², Merve Büyükbayram², \\ Emel Görgülü̈2 ${ }^{2}$ Nurten Özkan Zarif́ ${ }^{2}$ Ebru Yılmaz Keskin ${ }^{3 \oplus}$ \\ ${ }^{1}$ Department of Medical Genetics, Necip Fazl City Hospital, Kahramanmaraş; ${ }^{3}$ Division of Pediatric Hematology and Oncology, \\ ${ }^{2}$ Department of Pediatrics, Süleyman Demirel University Faculty of Medicine, Isparta, Turkey.
}

\begin{abstract}
Iron-refractory iron deficiency anemia (IRIDA) is an inherited iron metabolism disorder caused by mutations in TMPRSS6 gene encoding matriptase-2, which results in increased hepcidin synthesis. The hallmarks of the disease are hypochromic microcytic anemia, low transferrin saturation, slightly low or normal ferritin levels in contrast to classic iron deficiency anemia (IDA), inadequate response to oral iron, and only a partial response to parenteral iron. We report here a 6-year-old Syrian boy with unexplained microcytic anemia since one year of age. Genetic analysis of the TMPRSS6 gene revealed a novel homozygous nonsense mutation in exon 3 (c.234C>G; p.Y78* or p.Tyr78*). In the presence of hypochromic microcytic anemia accompanied by atypical iron parameters not in accordance with classic IDA, and inadequate response to iron therapy, IRIDA should be remembered in the differential diagnosis.
\end{abstract}

Key words: iron deficiency anemia, hepcidin, TMPRSS6, matriptase-2.

Iron deficiency anemia (IDA) has constituted a serious public health problem for centuries. It develops most commonly due to inadequate dietary intake. In the presence of hypochromic microcytic anemia, IDA is the first underlying cause to be considered. However, some inherited conditions with variable clinical characteristics may also result in microcytic anemia by causing defective iron metabolism.

Iron-refractory IDA (IRIDA) described about a decade ago is one of the inherited iron metabolism disorders, and develops due to lossof-function mutations in TMPRSS6 gene. ${ }^{1}$ As a result, affected individuals have inappropriately elevated hepcidin levels in contrast to classic IDA cases, whose serum hepcidin levels decrease markedly to promote intestinal iron

\footnotetext{
$凶 \quad$ Ebru Yılmaz Keskin

ebruyilmaz81@hotmail.com
}

Received 8th October 2018; revised 16th May 2019; accepted 5th July 2019 absorption. ${ }^{2,3}$ Remarkably, despite congenital, severe iron deficiency, affected cases display normal growth and intellectual development. ${ }^{2}$

We present here a child who was followed up for unexplained microcytic anemia since early childhood. Eventually, the case was diagnosed as IRIDA by genetic analysis which revealed a novel homozygous nonsense TMPRSS6 mutation.

\section{Case Report}

A 6-year-old Syrian male patient born to firstdegree cousin marriage was admitted due to microcytic anemia known since one year of age. He had history of inadequate response to oral iron therapy including the use of ferrous glycine sulfate ordered several times before by different physicians. Bone marrow aspiration studies had not identified the underlying cause, either. Although not proven, thalassemia intermedia had been thought in the differential diagnosis, and the case had been transfused twice. 
Among the family members, the 10-year-old brother also had microcytic anemia, and the mother had history of IDA during pregnancy.

At the time of admission to our clinic, the patient was using $2.5 \mathrm{mg} / \mathrm{kg} / \mathrm{d}$ iron (III)-hydroxide polymaltose complex for two weeks. Laboratory data of the patient and his available family members at the time of admission are presented in Table I. In addition, our case had negative tissue transglutaminase IgA and IgG antibodies examined for celiac disease accompanied by a normal serum IgA level, and his hemoglobin $(\mathrm{Hb})$ electrophoresis examination results were within normal range, $\mathrm{HbA0}$ level being $96.5 \%$.

In the presence of microcytic anemia for many years which was unresponsive to iron therapy, low transferrin saturation accompanied by normal serum ferritin level in the absence of an infection or an inflammatory condition, IRIDA was thought as the possible diagnosis.

For the purpose of confirming the diagnosis, TMPRSS6 gene sequence analysis of all coding exons and exon-intron boundaries was performed by next generation sequencing analysis (Miseq-Illumina Inc.). A novel homozygous mutation in exon 3 (c.234C>G; according to NM_153609.3) that causes a premature stop codon (p.Y78* or p.Tyr78*) was identified in the index case and his similarly affected sibling. In addition, the 15-year-old sister and the parents were found heterozygous for the same mutation. To validate the mutation in this family, we performed Sanger sequencing for the third exon of the TMPRSS6 gene in all the members of the family. Results of the Sanger sequencing analysis confirmed the variant we detected by next generation sequencing analysis (Fig. 1)

The identified nonsense mutation was neither found in Exome Aggregation Consortium nor 1000 Genomes databases. Prediction of the functional effect of the mutation was done by using in silico analysis tool MutationTaster (http://www.mutationtaster.org/). MutationTaster prediction was disease-causing. ${ }^{4}$ Variant was submitted to ClinVar; submission accession number is SCV000864243.1 (https:// www.ncbi. nlm.nih.gov/clinvar).

The patient received intravenous iron therapy. Response to treatment is summarized in Table II. This case report was written after obtaining the parents' written informed consent.

Table I. Laboratory findings of the patient and his available family members at the time of admission.

\begin{tabular}{|c|c|c|c|}
\hline & Proband & 15-year-old sister & 10-year-old brother \\
\hline Hemoglobin (g/L) & 7.2 & 13.7 & 8.9 \\
\hline Hematocrit (\%) & 26.2 & 40.4 & 29.7 \\
\hline Red blood cells $\left(10^{6} / \mu \mathrm{L}\right)$ & 5.25 & 5.35 & 5.91 \\
\hline MCV (fL) & 49.8 & 75.6 & 50.2 \\
\hline $\mathrm{MCH}(\mathrm{pg})$ & 13.8 & 25.6 & 15.0 \\
\hline $\mathrm{MCHC}(\mathrm{g} / \mathrm{dl})$ & 27.6 & 33.8 & 29.9 \\
\hline RDW (\%) & 21.5 & 16.2 & 21 \\
\hline White blood cells $\left(10^{3} / \mu \mathrm{L}\right)$ & 5.2 & 5.9 & 6.2 \\
\hline Platelets $\left(10^{3} / \mu \mathrm{L}\right)$ & 422 & 182 & 410 \\
\hline TSI (\%) & 6.7 & 23.2 & 6.2 \\
\hline Ferritin (ng/ml) & 37 & 10 & 51 \\
\hline C-reactive protein & Negative & Negative & Negative \\
\hline TMPRSS6 mutation & $\begin{array}{c}\text { c. } 234 \mathrm{C}>\mathrm{G}(\text { p.Y78*) } \\
\text { (p.Tyr78*) Homozygous }\end{array}$ & $\begin{array}{c}\text { c.234C>G (p.Y78*) } \\
\text { (p.Tyr78*) Heterozygous }\end{array}$ & $\begin{array}{c}\text { c.234C >G (p.Y78*) } \\
\text { (p.Tyr78*) Homozygous }\end{array}$ \\
\hline
\end{tabular}

MCV: mean corpuscular volume; $\mathrm{MCH}$ : mean corpuscular hemoglobin; MCHC: mean corpuscular hemoglobin concentration; RDW: red cell distribution width; TSI: transferrin saturation index. 


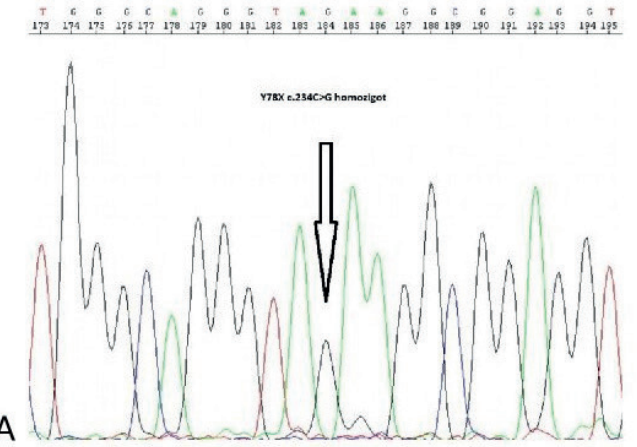

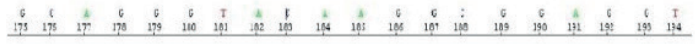

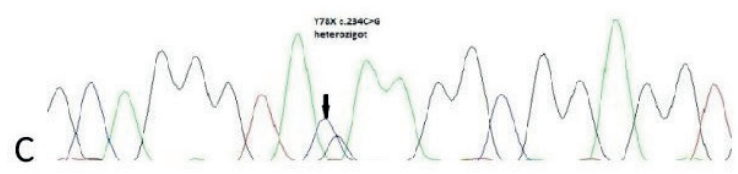

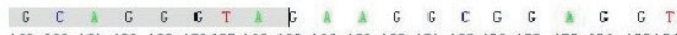

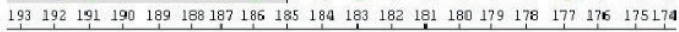

G : A A G G G G

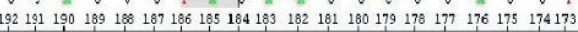

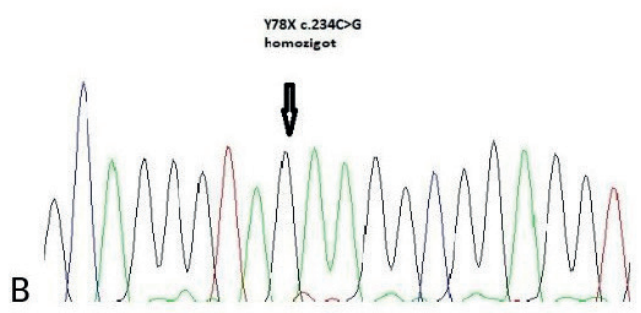

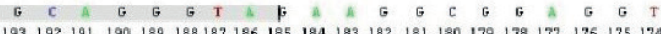

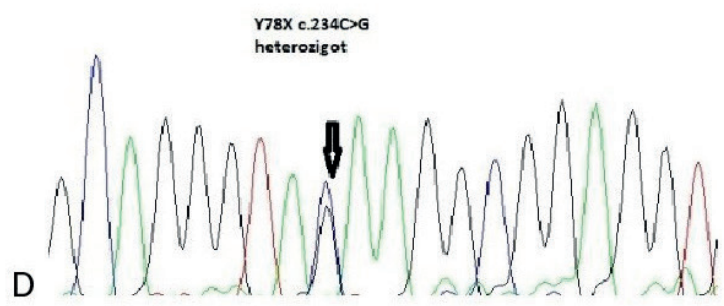

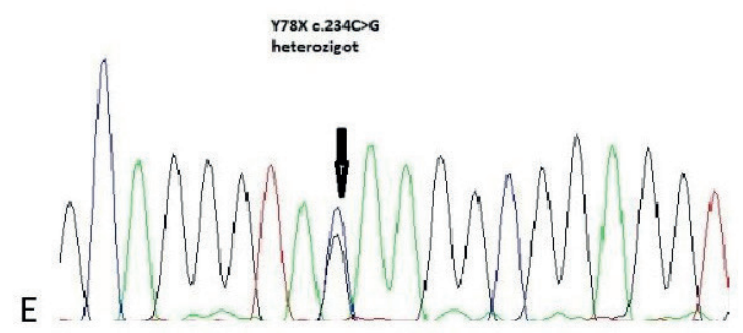

Fig. 1. Partial genome sequences of the TMPRSS6 gene of (A) the patient (B) the patient's affected brother (C) the father $(\mathrm{D})$ the mother $(\mathrm{E})$ the patient's unaffected sister.

\section{Discussion}

In a child with with hypochromic microcytic anemia, acquired IDA is the main disorder to be considered, whereas for congenital microcytic hypochromic anemias, the differential diagnosis is dominated by the thalassemia syndromes. Rarer forms of congenital microcytic anemias characterized by certain defects in iron transport, iron uptake, and mitochondrial iron utilization should also be included in the differential diagnosis. Among these are
IRIDA, divalent metal transporter 1 (DMT1) deficiency, congenital hypotransferrinemia, some hereditary forms of sideroblastic anemia, and aceruloplasminemia..$^{5}$ Although data regarding the prevalence of these rare congenital microcytic anemias are lacking, the recent increase in published IRIDA cases and affected families suggests that IRIDA may be the most common form.

IRIDA is an autosomal recessive disorder caused by mutations in the TMPRSS6 
Table II. Laboratory findings of the proband before and after intravenous (IV) iron replacement.

\begin{tabular}{lcccccc}
\hline & $\begin{array}{c}\text { Before IV } \\
\text { iron }\end{array}$ & $\begin{array}{c}\text { One week } \\
\text { after IV iron }\end{array}$ & $\begin{array}{c}\text { One month } \\
\text { after IV iron }\end{array}$ & $\begin{array}{c}\text { Two months } \\
\text { after IV iron }\end{array}$ & $\begin{array}{c}\text { Three months } \\
\text { after IV iron }\end{array}$ & $\begin{array}{c}\text { Five months } \\
\text { after IV iron }\end{array}$ \\
\hline Hemoglobin $(\mathrm{g} / \mathrm{dl})$ & 7.2 & 8.2 & 8.5 & 8.2 & 8.8 & 10.0 \\
Hematocrit $(\%)$ & 26.2 & 29.2 & 28.7 & 27.3 & 29.2 & 30.6 \\
Red blood cells $\left(10^{6} / \mu \mathrm{L}\right)$ & 5.25 & 5.62 & 5.36 & 5.05 & 5.23 & 5.78 \\
MCV (fL) & 49.8 & 52.0 & 53.5 & 54.1 & 55.8 & 53.0 \\
MCH (pg) & 13.8 & 14.5 & 15.9 & 16.1 & 16.9 & 17.3 \\
MCHC (g/dl) & 27.6 & 27.9 & 29.7 & 29.9 & 30.3 & 32.6 \\
RDW (\%) & 21.5 & 24.3 & 25.4 & 22.0 & 20.2 & 19.2 \\
TSI (\%) & 6.7 & n.a. & 5.2 & 1.7 & 3.6 & 2.5 \\
Ferritin (ng/ml) & 37 & 258 & 236 & 218 & 196 & 206 \\
\hline
\end{tabular}

$\mathrm{MCV}$ : mean corpuscular volume; $\mathrm{MCH}$ : mean corpuscular hemoglobin; $\mathrm{MCHC}$ : mean corpuscular hemoglobin concentration; RDW: red cell distribution width; TSI: transferrin saturation index; n.a.: not available.

gene which encodes matriptase-2, a type II transmembrane serine protease. ${ }^{1-3}$ TMPRSS6 gene is located on chromosome 22, contains 18 exons; all of the exons are coding. Matriptase- 2 contains a large extracellular region harbouring several structural domains. It is involved in downregulation of hepcidin expression, the master regulator of iron metabolism in hepatocytes.

In a previously untreated individual with hypochromic microcytic anemia, clues for an IRIDA diagnosis from the initial assessment of iron status include two patterns: 1) the degree of microcytosis (MCV 45-65 fL range) relative to the anemia ( $\mathrm{Hb} 6-8 \mathrm{~g} / \mathrm{dL}$ range); and (2) a markedly low serum iron level and low transferrin saturation index (usually $<5 \%$ ) in the presence of a slightly low or even normal ferritin. 2,6 At his first admission to us, our case had a moderate degree of anemia (Hb level, $7.2 \mathrm{~g} / \mathrm{dL}$ ) accompanied by profound microcytosis (MCV level, $49.8 \mathrm{fL}$ ), and his transferrin saturation level was low (6.7\%), while his ferritin level was within the normal range in the absence of a known infection or an inflammatory condition. In the presence of these findings, we strongly suspected IRIDA in our case.

Efficient intestinal iron absorption requires an acidic duodenal environment and a functioning duodenal epithelium. Common reasons for poor iron absorption include achlorhydria due to chronic proton pump inhibition and damage to the duodenum (eg, celiac sprue). ${ }^{7}$ Our case had no history of any drug use, and in his laboratory evaluation, antibodies associated with celiac disease were found to be negative. Inadequate response to oral iron therapy in the absence of simultaneous drug use or a disorder such as an inflammatory bowel disease further supported the diagnosis of IRIDA in our case.

Ideally, the plasma or urinary hepcidin levels could be measured to easily distinguish true iron deficiency from IRIDA. In IRIDA, normal-toelevated hepcidin levels are expected in contrast to classic IDA where hepcidin levels decrease to promote intestinal iron absorption. ${ }^{2}$ However, although more than a decade has passed since the discovery of hepcidin, there is yet no hepcidin assay approved by the Food and Drug Administration available for clinical use. Once a hepcidin assay becomes available, it may serve as a useful aid in the diagnosis of IRIDA. Our patient's hepcidin level was not measured due to technical equipment restrictions although we expect it to be in normal or above the normal range.

In a case suspected to have IRIDA with certain laboratory and clinical data mentioned above, the diagnosis of IRIDA is very likely especially 
if the iron deficiency truly appears to have its onset in infancy or childhood. ${ }^{2}$ The only current diagnostic test for IRIDA is sequencing of the TMPRSS6 gene. In our case, diagnosis of IRIDA was confirmed by mutational analysis.

Interestingly, in contrast to the usual form of IDA, IRIDA may not be associated with neuropsychological impairments as affected cases display normal growth and intellectual development. ${ }^{2,8}$ The potential role of TMPRSS6 in brain iron homeostasis was speculated, and it was hypothesized that inappropriately elevated hepcidin and normal to high ferritin levels compared with poor iron reserves may protect the brain in IRIDA. ${ }^{8}$

As expected in IRIDA cases, our patient displayed findings of defective iron utilization as evidenced by an elevation of ferritin levels after intravenous iron therapy with an only mild correction of his anemia. This observation may be explained by inappropriately elevated hepcidin levels in IRIDA patients. Hepcidin exerts its iron regulatory effects by binding to ferroportin which is the only known cellular iron exporter. It is expressed on the basolateral membrane of enterocytes, on the plasma membrane of macrophages and in hepatocytes. Since hepcidin binding leads to the internalization and degradation of ferroportin in lysosomes, dietary iron absorption and mobilization of iron from macrophage stores are decreased if hepcidin levels are elevated. ${ }^{2}$ Therefore, the decrease in ferroportin expression through hepcidin not only explains the development of iron deficiency, but also unresponsiveness to oral iron in IRIDA cases.

In heterozygous carriers of a TMPRSS6 mutation, iron deficiency was reported to develop under certain clinical conditions (such as in pregnancy), an observation inconsistent with recessive transmission. ${ }^{9}$ Similarly, TMPRSS6-haploinsufficient mice were observed to be more susceptible to iron deficiency under conditions of iron restriction or an increased iron requirement. ${ }^{10,11}$ Supporting these observations, the mother of our case had history of iron deficiency anemia during pregnancy which may be associated with her carrier status.

In few IRIDA cases followed-up until adulthood, increase in $\mathrm{Hb}$ levels to acceptable values was observed. ${ }^{12}$ This was explained by the consequence of the greater availability of the limited amount of dietary iron for erythropoiesis in adults affected by IRIDA than in pediatric cases who need iron also for body growth.

Premature termination codons that are followed by an intron that is located more than 50-55 nucleotides downstream are generally expected to trigger Nonsense-mediated mRNA decay (NMD). ${ }^{13}$ NMD is a mechanism that selectively eliminates mRNAs harboring premature termination codons. ${ }^{14}$ We report here a $C$ to $G$ transversion of the fifth base of exon 3 which causes a premature stop codon (p.Y78*). We expect that this mutation in our patient leads to no protein being translated at all and haploinsufficiency through NMD mechanism.

Up to now, at least 45 mutations including missense, nonsense, frameshifts and splicing defects in the TMPRSS6 gene leading to IRIDA phenotype have been reported in the literature. ${ }^{2}$ To the best of our knowledge, only six nonsense mutations have been reported in TMPRSS6 gene so far: p.Y355*, p.Y393*, p.S561*, p.R599*, p.Q405* and p.K752*.15 The variation we detected in our patient (c.234C>G; according to NM_153609.3), is a nonsense mutation which has not been reported before.

De Falco et al. ${ }^{16}$, stated that patients harboring two nonsense mutations present a more severe anemia and microcytosis compared with the patients carrying either 2 missense mutations or 1 missense and 1 nonsense mutation. Our patient's $\mathrm{Hb}$ level and mean corpuscular volume (MCV) of his erythrocytes at admission were $7.2 \mathrm{~g} / \mathrm{dL}$ and $49.8 \mathrm{fL}$, respectively, while his 10year old brother had an $\mathrm{Hb}$ of $8.9 \mathrm{~g} / \mathrm{dL}$ and an MCV of $50.2 \mathrm{fL}$. Anemia of the two siblings may have been even more severe during their early 
childhood, a time period when iron requirement for body growth is more marked.

In conclusion, we report a novel homozygous nonsense mutation in TMPRSS6 gene in a 6-yearold Syrian boy and his sibling with unexplained microcytic anemia before. Through the timely genetic diagnosis of this disorder with a quite favorable prognosis, unnecessary (invasive) examinations can be avoided. Our results expand the mutation spectrum of the TMPRSS6 gene contributing new data on genotypephenotype correlations in IRIDA. Further studies are required to better understand the effect of TMPRSS6 gene alterations on the phenotype and protein function.

\section{Acknowledgments}

The authors thank Serdar Ceylaner from Intergen Genetic Diagnosis Center for assisting with molecular genetic analysis.

\section{REFERENCES}

1. Finberg KE, Heeney MM, Campagna DR, et al. Mutations in TMPRSS6 cause iron-refractory iron deficiency anemia (IRIDA). Nat Genet 2008; 40: 569571.

2. Heeney MM, Finberg KE. Iron-refractory iron deficiency anemia (IRIDA). Hematol Oncol Clin North Am 2014; 28: 637-652.

3. Shokrgozar N, Golafshan HA. Molecular perspective of iron uptake, related diseases, and treatments. Blood Res 2019; 54: 10-16.

4. Schwarz JM, Cooper DN, Schuelke M, Seelow D. Mutation Taster2: mutation prediction for the deepsequencing age. Nat Methods 2014; 11: 361-362.

5. Camaschella C. How I manage patients with atypical microcytic anaemia. Br J Haematol 2013; 160: 12-24.
6. Brissot $\mathrm{P}$, Bernard DG, Brissot E, Loréal O, Troadec MB. Rare anemias due to genetic iron metabolism defects. Mutat Res 2018; 777: 52-63.

7. Azad SM, Kapoor R, Bannerji R, Ray J, Mitra M. Celiac disease masquerading as refractory iron deficiency anemia. Int J Contemp Pediatr 2017; 4: 672-673.

8. Arsenault V, Mailloux C, Bonnefoy A, Lemyre E, Pastore Y. Iron-refractory iron deficiency anemia may not lead to neurocognitive dysfunction: a case report. Pediatrics 2016; 138: e20153608.

9. Yilmaz Keskin E, Yenicesu I. Iron-refractory iron deficiency anemia. Turk J Haematol 2015; 32: 1-14.

10. Nai A, Pagani A, Silvestri L, Camaschella C. Increased susceptibility to iron deficiency of Tmprss6-haploinsufficient mice. Blood 2010; 116: 851-852. 57.

11. Finberg KE, Whittlesey RL, Fleming MD, Andrews NC. Down-regulation of Bmp/Smad signaling by Tmprss 6 is required for maintenance of systemic iron homeostasis. Blood 2010; 115: 3817-3826.

12. Melis MA, Cau M, Congiu R, et al. A mutation in the TMPRSS6 gene, encoding a transmembrane serine protease that suppresses hepcidin production, in familial iron deficiency anemia refractory to oral iron. Haematologica 2008; 93: 1473-1479.

13. Maquat LE. Nonsense-mediated mRNA decay: splicing, translation and mRNP dynamics. Nat Rev Mol Cell Biol 2004; 5: 89-99.

14. Chang YF, Imam JS, Wilkinson MF. The nonsensemediated decay RNA surveillance pathway. Annu Rev Biochem 2007; 76: 51-74.

15. De Falco L, Silvestri L, Kannengiesser C, et al Functional and clinical impact of novel TMPRSS6 variants in iron-refractory iron-deficiency anemia patients and genotype-phenotype studies. Hum Mutat 2014; 35: 1321-1329.

16. De Falco L, Sanchez M, Silvestri L et al. Iron refractory iron deficiency anemia. Haematologica 2013; 98: 845-853. 